\title{
Coronary artery disease associated with severe mitral and tricuspid valve regurgitation after left pneumonectomy: report of a successful hybrid procedure
}

\author{
Marco Gennaria, ${ }^{\mathrm{a}}$, Samer Kassema ${ }^{\mathrm{a}}$, Giovanni Teruzzi ${ }^{\mathrm{b}}$ and Marco Agrifoglio ${ }^{\mathrm{a}}$ \\ a Department of Cardiovascular Disease, Cardiac Surgery Unit II, IRCCS Centro Cardiologico Monzino, Milan, Italy \\ b Interventional Cardiology Unit, IRCCS Centro Cardiologico Monzino, Milan, Italy \\ * Corresponding author. Department of Cardiovascular Disease, Cardiac Surgery Unit, IRCCS Centro Cardiologico Fondazione Monzino, Via Parea 4, Milan, Italy \\ Tel: +39-328 8286140; e-mail: marcogennari.md@gmail.com (M. Gennari).
}

Received 30 October 2013; received in revised form 18 March 2014; accepted 26 March 2014

\begin{abstract}
The literature concerning heart surgery after pneumonectomy is still poor. Moreover, there is still a lack of a standardized approach to such a patient in the decision-making process. Here, we report a case of a patient who had previously had left pneumonectomy for malignancy and who had coronary artery disease and mitral and tricuspid regurgitation treated with a hybrid procedure.
\end{abstract}

Keywords: Pneumonectomy $\cdot$ Heart valve $\cdot$ Coronary stents $•$ Percutaneous coronary intervention

\section{INTRODUCTION}

Concomitant heart valve surgery and coronary artery revascularization after previous pneumonectomy poses challenges for the surgical approach. The literature concerning coronary artery bypass grafting $(\mathrm{CABG})$ and valvular operations following pneumonectomy reports only a few cases, with a substantial lack of official guidelines for the best management [1].

Here, we report a successful hybrid procedure for concomitant coronary artery and valve disease in a patient with prior pneumonectomy.

\section{CASE REPORT}

A 71-year-old man was referred to hospital for severe mitral and tricuspid regurgitation 4 years after left pneumonectomy for a lung cancer. The chest X-ray revealed marked rotation and displacement of the heart shadow into the left pleural space and compensatory hyperinflation of the right lung. Pulmonary functional tests revealed a forced expiratory volume in $1 \mathrm{~s}$ (FEV1) of $2.02 \mathrm{I}$ (53\% predicted) and a forced vital capacity (FVC) of 1.51 I (54\% predicted). The transthoracic echocardiogram showed severe mitral valve regurgitation because of a prolapse of the posterior leaflet and important regurgitation of the tricuspid valve with preserved left ventricular ejection fraction. The preoperative coronary angiography showed $75 \%$ stenosis of the left anterior descending artery (LAD) and $80 \%$ stenosis of the left circumflex artery (LCX).

The chest 64-slice computed tomography (CT) confirmed the marked leftward displacement of the heart. It also showed that the LAD would not be easily accessible by median sternotomy and that the LCX was close to the vertebral column and was accessible at best only by left posterolateral thoracothomy (Fig. 1A and B).
Given these anatomical findings, we planned a hybrid procedure. The myocardial revascularization was performed with percutaneous coronary intervention (PCI) and stenting of the LAD and LCX with bare metal stents, with good angiographic results.

A month later, a median sternotomy was performed. The pericardial sac was opened and the heart was displaced to the left and rotated around its long axis, which brought both the right and left atrium more into view. Standard cannulation for cardiopulmonary bypass was achieved by inserting an arterial cannula into the ascending aorta and two venous cannulas into the cava veins. Mitral valve repair was easily performed with middle quadrangular resection of the P2 scallop of the posterior leaflet and positioning of a 30-mm Carpentier-Edwards Physio Ring (Irvine, CA, USA) associated with the tricuspid valve repair by positioning a $28-\mathrm{mm}$ Cosgrove-Edwards Ring (Irvine, CA, USA). The postoperative course was uneventful. The transthoracic echocardiogram showed absence of residual regurgitation of the valves.

A cardiac CT showed good patency of coronary stents (Fig. 1C and $\mathrm{D}$ ) and the right localization of the mitral and tricuspid prosthetic rings (Fig. 2). The patient was discharged home on the 11th postoperative day.

\section{DISCUSSION}

Previous pneumonectomy raises several issues concerning the best approach for cardiac surgery, especially regarding exposure, line placement for cardiopulmonary bypass, choice of conduits and preservation of respiratory function [2].

Noteworthy is the surgical approach: CABG seems technically easier to perform by standard median sternotomy in patients with prior right pneumonectomy, because of the position of the heart, 

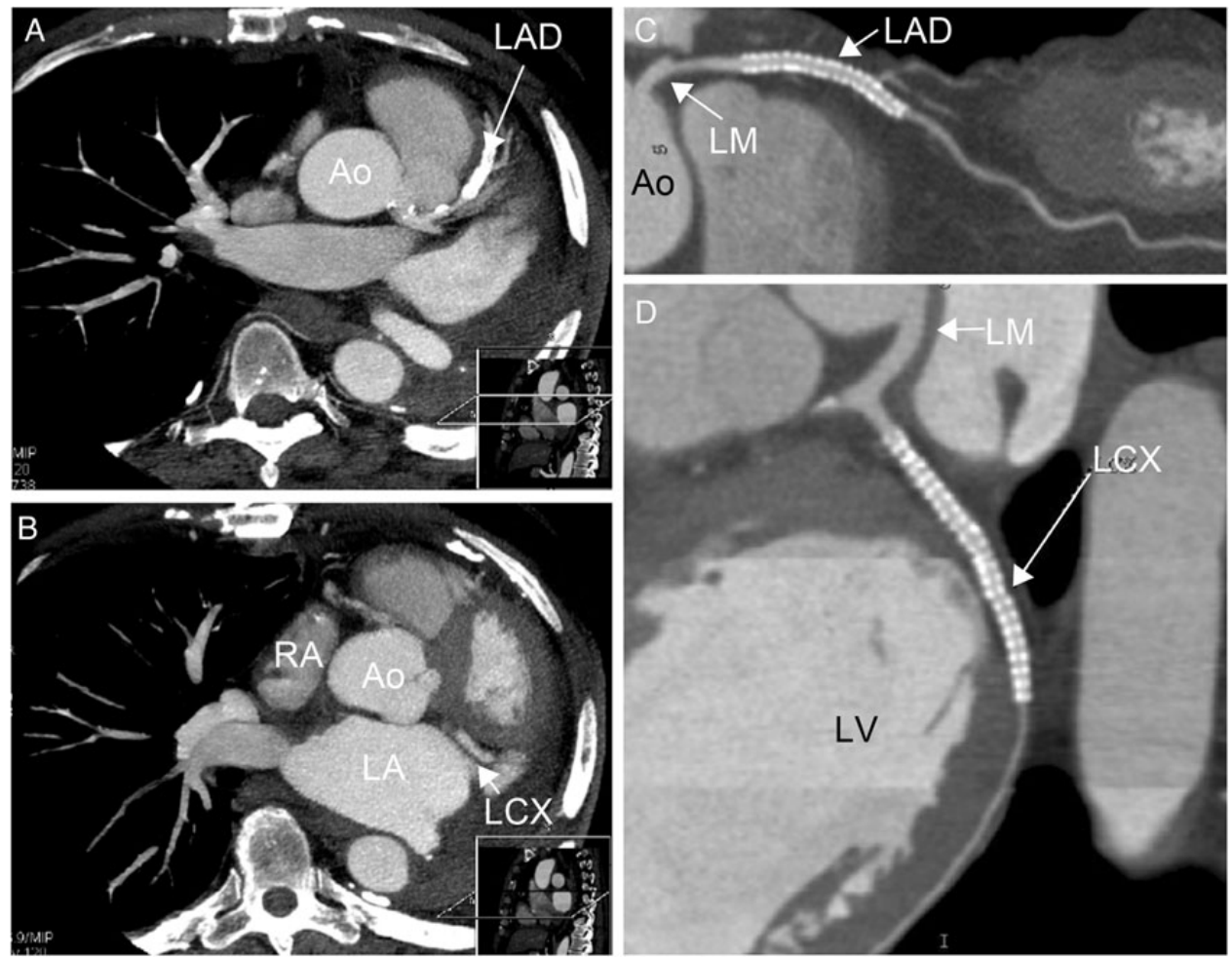

Figure 1: Multiplanar reconstruction of heart chambers and coronary arteries. CT showed the marked leftward displacement of the heart, that the left anterior descending artery (LAD) would not be so easily accessible by median sternotomy and that the left circumflex artery (LCX) was close to the spine and deemed accessible only by posterolateral left thoracothomy (A and B). The CT post-PCl showed the patency and no evidence of in-stent restenosis of LAD (C) and LCX (D). Ao: ascending aorta; LA: left atrium; LV: left ventricle; RA: right atrium; LM: left main artery.
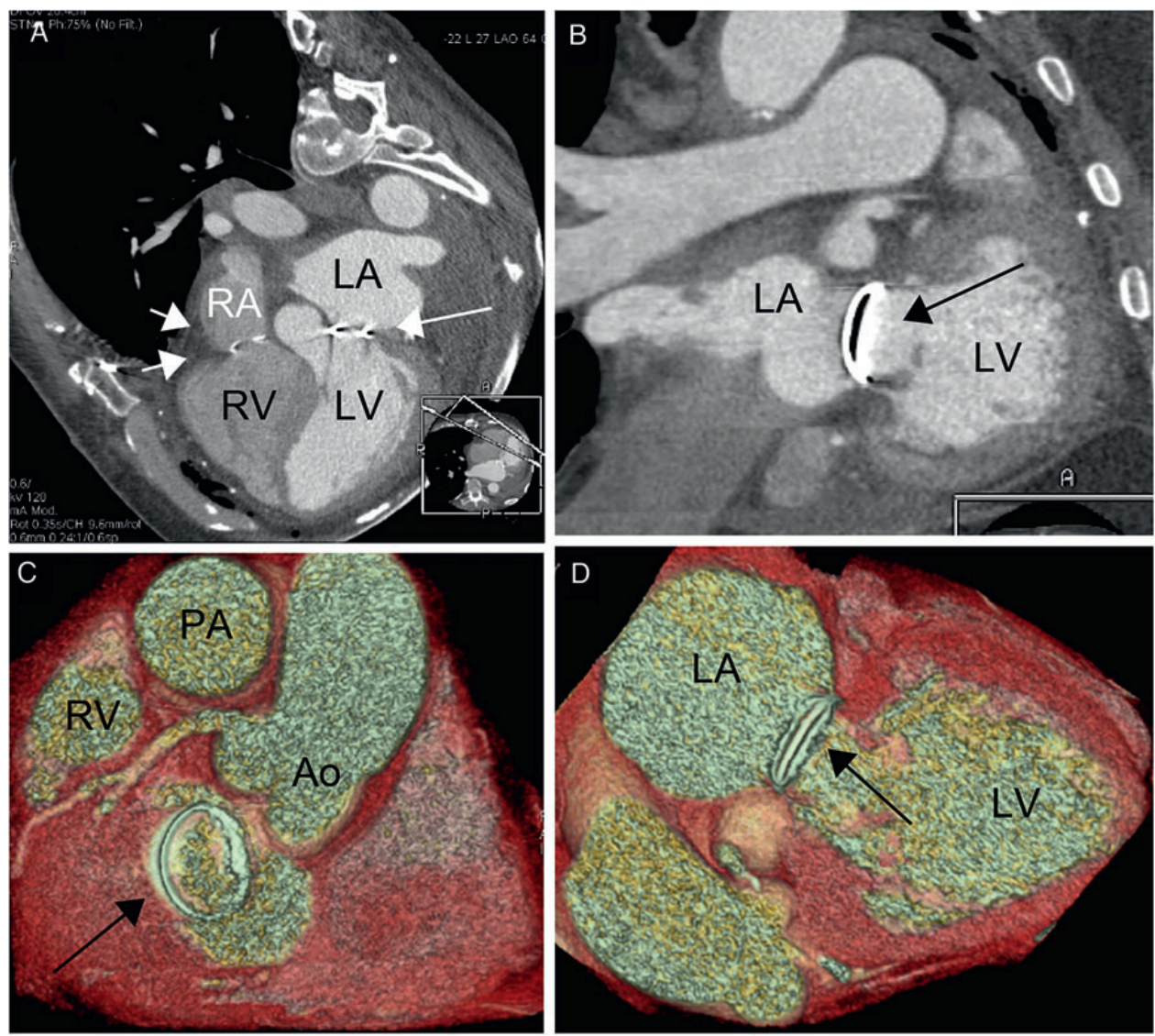

Figure 2: Multiplanar ( $\mathbf{A}$ and $\mathbf{B}$ ) and volume rendering ( $\mathbf{C}$ and $\mathbf{D}$ ) reconstruction of heart chambers. CT showed the localization of the mitral (arrows) and tricuspid (small arrows) prosthetic rings. Ao: ascending aorta; PA: pulmonary artery; LA: left atrium; LV: left ventricle; RA: right atrium; RV: right ventricle. 
whereas mitral valve exposure is sometimes difficult to obtain [2-4]. Furthermore, prior left pneumonectomy causes difficult access to the coronary arteries by median sternotomy and some authors advise posterolateral left thoracothomy for CABG operations in this setting $[3,5]$.

Another consideration is the ability to establish cardiopulmonary bypass (CPB) because of possible displacement of the arterial and venous structures. Accordantly, axillary and femoral sites have to be considered in advance.

The choice of the conduits for CABG is still debated. In particular, the use of the internal thoracic artery is controversial because of technical difficulty in its harvesting and the possible mismatch between its length and the displaced vessel to be bypassed.

Last, but not the least, special attention must to be paid to preserving the respiratory function. Given that respiratory distress is the most frequent postoperative complication in such patients, every effort must be made to reduce this possibility; actual strategies comprise preoperative physiotherapy, use of bronchodilators, steroids and antibiotics, and the recourse to off-pump coronary artery bypass (OPCAB), which reduces the systemic inflammatory response to $\mathrm{CPB}$ and, thus, the possibility of $\mathrm{CPB}$ induced pulmonary complications.

Preoperative chest CT or magnetic resonance scans are essential to plan correct surgical strategy.

The hybrid procedure is an unorthodox and staged approach after prior pneumonectomy; it has been reported previously, only for coronary disease and never for concomitant valvular and coronary diseases. Our hybrid ( $\mathrm{PCl}$ followed by valve surgery) strategy enabled us to treat both diseases successfully in this unusual and technically demanding patient. The new resources of transcatheter aortic valve implantation and Mitra-Clip ${ }^{\circledR}$ are also an attractive possibility for the near future to avoid open surgery in pneumonectomized patients.

\section{Funding}

This work was supported by Abbott Laboratories, Illinois, USA.

Conflict of interest: none declared.

\section{REFERENCES}

[1] Fragkidis A, Dimitriou A, Dougenis D. Coronary artery bypass grafting and/ or valvular surgery in patients with previous pneumonectomy. J Cardiothorac Surg 2012;7:110.

[2] Stoller JK, Blackstone E, Pettersson G, Mihaljevic T. Coronary artery bypass graft and/or valvular operations following prior pneumonectomy: report of four new patients and review of the literature. Chest 2007;132:295-301.

[3] Shanker VR, Yadav S, Hodge AJ. Coronary artery bypass grafting with valvular heart surgery after pneumonectomy. ANZJ Surg 2005;75:88-90.

[4] Izzat MB, Regragui IA, Angelini GD. Mitral valve replacement after previous right pneumonectomy. Ann Thorac Surg 1995;59:222-4.

[5] El-Hamamsy I, Stevens LM, Perrault LP, Carrier M. Right pneumonectomy and thoracoplasty followed by coronary artery bypass grafting and mitral valve replacement. J Thorac Cardiovasc Surg 2003;125:215-6. 